\title{
Green tea for the prevention of cancer: evidence of field epidemiology
}

\author{
Min Zhang*, Lin Li, Ping Liu, C. D’Arcy J. Holman
}

School of Population Health, The University of Western Australia, Perth, WA, Australia

*Corresponding author: Min Zhang, MD, PhD, Director of Lu Cha Sino-Australian Research Collaboration, School of Population Health, The University of Western Australia, 35 Stirling Highway, Crawley, Perth, WA 6009, Australia

Submission date: August 16, 2012, Acceptance date: October 13, 2012; Publication date:

October 15, 2012

\begin{abstract}
$\underline{\text { Abstract }}$
Background: Tea is derived from the leaf of Camellia sinensis, a natural beverage widely consumed around the world. Geological and botanical evidence suggests that the tea plant originated from China. Varying methods of processing tea leaves lead to green tea, black tea, or Oolong tea, which differ in their concentrations of polyphenols. Green tea polyphenols appear to have anti-tumorigenic properties, and form 30-40\% of the dry weight of green tea compared with only $3-10 \%$ of black tea. Numerous studies in multiple animal models and different cancer cell lines have demonstrated the anti-tumorigenesis by green tea polyphenols. Despite the consistency of laboratory results, evidence of this effect occuring in humans has been inconclusive to date.
\end{abstract}

Objective: To investigate if green tea consumption was associated with longer survival rates in ovarian cancer patients, and a lower risk of ovarian, breast, and colorectal cancer, in addition to adult leukemia

Methods: We have conducted one prospective cohort study in ovarian cancer patients, and five case-control studies in ovarian, breast, and colorectal cancers, and leukemia over the past decade. Tea consumption was measured using a structured questionnaire by face-to-face interviews. The validity and reliability of the questionnaire was assessed in a preliminary study, and then evaluated by a test-retest. Cox proportional hazards regression models were used to obtain hazard ratios(HRs), 95\% confidence intervals(95\% CIs), and were adjusted for age at diagnosis, locality, body mass index(BMI), parity, International Federation of Gynecology and Obstetrics (IFGO) stage, histologic grade of differentiation, cytology of ascites, residual tumour, and chemotherapeutic status. Odds ratios(ORs) and 95\% CIs were obtained using logistic regression analyses, which accounted for demographic, lifestyle, hormonal and family cancer factors, and potential confounders. 
Results: Higher green tea consumption was consistently observed as being associated with a lower risk of mortality due to ovarian cancer, and a decreased risk of ovarian, breast, and colorectal cancers, and adult leukemia occurrences in our observational studies. The adjusted HR and $95 \% \mathrm{CI}$ for case mortality from ovarian cancer was $0.40(0.18-0.90)$ in the patients who consumed green tea at the highest level compared with non-tea drinkers. Compared with never or seldom tea drinkers, the adjusted ORs ranged from 0.07 to 0.61 for ovarian, breast, and colorectal cancers, and adult leukemia in those who consumed green tea at the highest level. Significant inverse dose-response relationships were also observed for quantity, duration, and frequency of green tea consumed.

Conclusion: We concluded that regular consumption of green tea enhanced survival of ovarian cancer, and decreased risks of ovarian, breast, and colorectal cancers, and adult leukemia. Evidence from our observational studies supported the protective effect of green tea against cancers, and this evidence will provide a knowledge platform from which to launch interventional studies for cancer prevention in the next stage.

Key words: Green tea, nutrition epidemiology, case-control studies, cohort studies, risk factor, cancer survival, breast cancer, colorectal cancer, adult leukemia, ovarian cancer

\section{INTRODUCTION:}

Tea, derived from the leaf of Camellia sinensis, is one of the most frequently consumed beverages in the world after water. Varying methods of processing tea leaves lead to green tea, black tea or Oolong tea, which differ in their concentrations of polyphenols[1,2]. The polyphenols include (-)-epicatechin(EC), (-)-epigallocatechin(EGC), (-)-epicatechin-3-gallate (ECG), and (-)-epigallocatechin-3-gallate(EGCG), with EGCG being the major polyphenol in tea and other polyphenols that are chemically related to EGCG [1]. If the leaves are immediately heated, then the enzymes that oxidise polyphenols are inactivated, and the result is green tea. If the leaves are crushed and allowed to undergo enzyme-mediated oxidation('fermentation'), much of the indigenous polyphenols are converted to thearubigins and theaflavins, which are responsible for the characteristic colour and taste of black tea. Oolong tea results from partially oxidised tea leaves [1,2].

The three types of tea have different concentrations of polyphenols. Tea polyphenols form $30-40 \%$ of the dry weight of green tea compared with only 3-10\% of black tea[2]. A typical green tea beverage contains $240-320 \mathrm{mg}$ of tea polyphenols. Over 90 bioavailability studies have conclusively demonstrated that plasma concentrations of EGCG, EGC and EC reach peak levels 1.5-2.5 hours after ingestion, and that urinary excretion is mostly complete within 8-9 hours [3]. Cumulative excretion of tea polyphenols escalate by increasing the intake of green tea in humans [4]. Animal studies have demonstrated that tea polyphenols absorbed from the gastro-intestinal tract are widely distributed in body organs[5]. An increase in the antioxidant capacity of plasma occurs following ingestion of green tea in humans[6], as does a decrease in plasma and urinary 8-hydroxydeoxyguanosine, a marker of DNA oxidative damage by reactive oxygen species[6-8]. 
As a natural and historic beverage, tea is consumed worldwide, although at greatly varying levels and practices[2]. In western countries, the dominant type of tea consumed is black tea. Green tea, the main tea beverage in Japan and many parts of China, accounts for about $20 \%$ of worldwide tea production. Oolong tea, consumed mainly in southern China and Taiwan[1], accounts for less than $2 \%$ of tea production. Tea is now grown in about 30 countries, but geological and botanical evidence suggests that the tea plant originated from China[1]. As one of the most ancient and common beverages, tea has been consumed for thousands years in the orient, and plays a central part in Chinese culture, with tea-drinking practices specific to different areas including the southeast and northeast regions of China.

For over three decades [9], green tea has attracted increasing attention for its purported health benefits, including anti-cancer effects. Numerous studies in multiple animal models and different cancer cell lines have demonstrated anti-tumorigenesis by green tea polyphenols[10,11]. Despite the consistency of laboratory results, evidence to date in humans has been mixed. In light of the variations in tea drinking practices, and inconclusive results from epidemiological studies, the research team performed one cohort study, and five case-control studies since 1999. These studies, conducted in southeast and northeast China, investigated whether green tea consumption was associated with longer survival rates in ovarian cancer patients, and a lower risk of incidence of ovarian, breast, and colorectal cancers, and adult leukemia. The associations between the malignancies and tea consumption were assessed by measuring type, duration, frequency, quantity of tea, and the interactions between tea consumption and other lifestyle factors.

\section{METHODS:}

The methods used in the prospective cohort study were reported elsewhere[12]. The methods for five case-control studies were similar, and details of these observational studies were published elsewhere[13-17]. All the participating university hospitals were public hospitals in Hangzhou and Shenyang, the capital cities of the Zhejiang and Liaoning Province respectively, with 5002,250 beds, and received patients from across the provinces. The participants, aged 18 to 87 years, were Chinese people who resided in the provinces. Cases were identified from medical records in participating hospitals, and all diagnoses were histopathologically confirmed after surgery, or based on hematological standards. The patients were excluded if their cancer or leukemia was neither the primary nor final diagnosis. All relevant hospital and laboratory pathology reports were reviewed daily to ensure the completion of recruitment. The proportions of lost or non-responding patients among the cases ranged from $0.2 \%$ to $1.8 \%$ across the studies. During the same period of data collection, community controls were randomly selected from residents with the assistance of local community councils. Hospital controls without malignant disease were randomly and consecutively selected from inpatients, e.g. orthopedics wards, or outpatients in the same participating hospitals. These cases were matched by age, gender, and living area, using a daily update of the list of cases. Each control that consented to participate was recruited as the first within the matching criteria to attend the same participating hospital. Potential controls were excluded if they had a previous diagnosis of cancer, leukemia, or other malignant disease. The proportions of selected controls that participated in our studies were 90.0- 
98.8\%. The projects received approvals from the Chinese hospital authorities, and from the Human Research Ethics Committee of Australian universities at which we were based.

Subjects were briefed regarding the general aims of the study to investigate lifestyle factors, confidentiality, and anonymity issues. An appointment for interview was made after obtaining their consent at initial contact. A face-to-face interview, usually lasting 30-40 minutes, was then conducted in the hospital setting using a structured questionnaire. The cases were interviewed in their wards, most of them within three months after diagnosis, while the controls were interviewed at the same hospitals, or in the communities in which they resided. A validated and reliable questionnaire was used to collect the information on: (a) demographic and lifestyle characteristics, e.g. residential area, education, smoking, alcohol consumption, and physical activity; (b) tea consumption; (c) food consumption assessed by a 100-item food frequency questionnaire; and (d) factors relevant to hormonal status and family history of cancers[18-20]. The cut-off date for dietary intake and physical activity was set as one year prior to diagnosis (cases) or interview(controls). If there were any changes in their habits recently, only information on the habits before the change was used in data analysis. The questionnaire was translated into Chinese, and re-checked after back-translation by professional Chinese translators.

Tea consumption was measured using a tea questionnaire. The reproducibility of the tea questionnaire was evaluated, and the intraclass correlation coefficient was 0.83 for tea consumption[20]. This high coefficient for test-retest reliability suggested that the questionnaire may be relied upon in assessing tea consumption. This self-reported instrument was used to assess several aspects of tea consumption. The participants were first classified as either 'never' or 'ever' tea drinkers in their lives. Information was then sought from all 'ever' drinkers on their consumption patterns, preparation methods, type of tea drank, duration of each type of tea drinking, usual frequency of cups consumed(counting the number of 350-400ml cups, the typical teacup used by residents in southeast and northeast China), frequency of new batches of tea brewed, quantity of dried tea-leaf consumed per year, and date that tea drinking ceased. The frequency of cups and number of new batch of tea consumed were categorised as: never or seldom, once a month, 2-3 times a month, once a week, 2-3 times a week, 4-6 times a week, once a day, 2-3 times a day and $\geq 4$ times a day. For infrequent tea drinkers, and those drinking less than one cup at a time, the actual tea consumption was recorded. The quantity of dried tea leaves consumed per year was also requested in terms of a standard Chinese measure, the liang (equivalent to 50 grams).

All data was checked for completeness at the end of each interview. The data was coded and analyzed using the SPSS version 19.0. Data collected by different interviewers was compared, and confirmed no consistent difference in the recording of key variables such as tea consumption within cases or controls. The tea consumption variables were grouped into three or five categories to facilitate analysis with non-tea drinkers as the reference group. Demographic characteristics and potential risk factors between cases and controls were compared using a $t$-test for continuous variables, and Chi-square test for categorical variables. Cox proportional hazards regression models were used to obtain HRs and 95\% CIs, adjusting for age at diagnosis, locality, BMI, parity, FIGO stage, histologic grade of differentiation, cytology of ascites, residual tumour, 
and chemotherapeutic status. ORs and their corresponding 95\% CIs and p-values for potential risk factors, were obtained using logistic regression analyses, accounting for demographic, lifestyle, hormonal, and family cancer factors, and potential confounders. Univariate analysis was also undertaken to screen potential explanatory variables for subsequent multivariate analysis. Each quantitative or ordinal measure of tea consumption was subjected to a linear trend test. The studies only focused on green tea, because too few tea drinkers drank only black or Oolong tea.

\section{RESULTS:}

A total of 6308 participants were recruited to the six studies. Four studies were conducted in southeast China, i.e. a cohort $\operatorname{study}(n=254)$ and a case-control study $(n=906)$ on ovarian cancer, a case-control study $(n=2018)$ on breast cancer, and a case-control study $(n=217)$ on adult leukemia. Two studies were conducted in northeast China, i.e. a case-control study $(n=1233)$ on breast cancer, and another three parallel case-control studies $(n=1680)$ on breast and colorectal cancer, and adult leukemia.

Table 1. Selected characteristic of participants in breast cancer studies in southeast China 20042005 and northeast China 2008-2009

\begin{tabular}{|c|c|c|}
\hline Selected characteristics & $\begin{array}{c}\text { Southeast } \\
\text { participants }(n=2018)\end{array}$ & $\begin{array}{l}\text { Northeast participants } \\
(n=1233)\end{array}$ \\
\hline Age at interview (years) & $48.4 \pm 10.4$ & $49.2 \pm 9.5$ \\
\hline$<40$ & $357(17.7)$ & $185(15.0)$ \\
\hline $40-49$ & $865(42.9)$ & $490(39.7)$ \\
\hline $50-59$ & $524(26.0)$ & $405(32.8)$ \\
\hline $60-69$ & $188(9.3)$ & $108(8.8)$ \\
\hline$\geq 70$ & $84(4.2)$ & $45(3.6)$ \\
\hline \multicolumn{3}{|l|}{ Education } \\
\hline No formal education & $334(16.6)$ & $20(1.6)$ \\
\hline Primary & $579(28.7)$ & $145(11.8)$ \\
\hline Secondary & $771(38.2)$ & $777(63.0)$ \\
\hline Tertiary & $334(16.6)$ & $291(23.6)$ \\
\hline Resident in rural area & $946(46.9)$ & $87(7.1)$ \\
\hline BMI (5 years ago, $\mathrm{kg} / \mathrm{m}^{2}$ ) & $22.6 \pm 3.1$ & $23.1 \pm 2.9$ \\
\hline$<25$ & $1635(81.0)$ & $953(77.6)$ \\
\hline$\geq 25$ & $383(19.0)$ & $275(22.4)$ \\
\hline Tobacco smoking & $34(1.7)$ & $78(6.3)$ \\
\hline Passive smoking & $1176(58.3)$ & $685(55.6)$ \\
\hline Alcohol consumption & $828(41.0)$ & $370(30.0)$ \\
\hline \multicolumn{3}{|l|}{ Tea consumption } \\
\hline No & $906(44.9)$ & $911(73.9)$ \\
\hline Yes & $1112(55.1)$ & $322(26.1)$ \\
\hline \multicolumn{3}{|l|}{ Type of tea consumption } \\
\hline Green tea & $990(89.0)$ & $205(63.7)$ \\
\hline Black tea & $54(4.9)$ & $30(9.3)$ \\
\hline Oolong tea & $3(0.3)$ & $4(1.2)$ \\
\hline
\end{tabular}


Green and black tea
Physical activity (MET hrs/week)
Energy intake (kilocalories/day)
$65(5.8)$

$60.5 \pm 65.1$

$2134.2 \pm 540.9$
$83(25.7)$

$85.5 \pm 77.0$

$2413.1 \pm 639.3$

Values expressed as mean \pm SD or number (percent).

Table 1 presents the selected characteristics of participants in breast cancer studies conducted in southeast China in 2004-2005, and northeast China in 2008-2009. There were sizable variations in demographic characteristics between the participants in the two studies, including education, living in rural area, tobacco smoking, alcohol drinking, physical activity(weekly MET-hour), and energy intake. In particular, there were differences in tea consumption between Chinese women in southeast and northeast China. Some $55.1 \%$ of Chinese women in the southeast were tea drinkers, compared with $26.1 \%$ of their counterparts in northeast China. Among the tea drinkers, $89 \%$ and $61 \%$ drank green tea only, $4.9 \%$ and $9.3 \%$ black tea only, $0.3 \%$ and $1.2 \%$ Oolong tea only, and $5.8 \%$ and $25.7 \%$ drank both green and black tea.

Table 2. Observational studies in green tea and cancer with the Lu Cha (green tea) SinoAustralian Research Collaboration

\begin{tabular}{|c|c|c|c|c|}
\hline $\begin{array}{l}\text { Location/year } \\
\text { commenced }\end{array}$ & Cancer & Participants & $\begin{array}{l}\text { Lowest vs. highest level } \\
\text { of green tea exposure }\end{array}$ & $\begin{array}{c}\text { OR or HR (95\% } \\
\text { CI) } \\
\text { for highest vs. } \\
\text { lowest level }\end{array}$ \\
\hline \multicolumn{5}{|l|}{ Cohort studies } \\
\hline \multicolumn{5}{|l|}{ Southeast China } \\
\hline 2003 [12] & Ovarian & 254 & $\begin{array}{l}\text { None vs. } \geq 500 \mathrm{~g} \text { dried } \\
\text { tealeaf/yr }\end{array}$ & $0.40(0.18-0.90)$ \\
\hline \multicolumn{5}{|l|}{ Case-control studies } \\
\hline \multicolumn{5}{|l|}{ Southeast China } \\
\hline 1999 [13] & Ovarian & 906 & None vs. $\geq 1$ cups/day & $0.43(0.30-0.63)$ \\
\hline 2004 [14] & Breast & 2018 & $\begin{array}{l}\text { None vs. } \geq 750 \mathrm{~g} \text { dried } \\
\text { tealeaf/yr }\end{array}$ & $0.61(0.48-0.78)$ \\
\hline 2005 [15] & Leukemia & 217 & $\begin{array}{l}\text { None vs. } \geq 1001 \mathrm{~g} \text { dried } \\
\text { tealeaf/yr }\end{array}$ & $0.39(0.17-0.91)$ \\
\hline \multicolumn{5}{|l|}{ Northeast China } \\
\hline $2007[16]$ & Breast & 1233 & $\begin{array}{l}\text { None vs. } \geq 750 \mathrm{~g} \text { dried } \\
\text { tealeaf } / \mathrm{yr}\end{array}$ & $0.43(0.21-0.91)$ \\
\hline 2009 [17] & Breast & 885 & $\begin{array}{l}\text { None vs. } \geq 750 \mathrm{~g} \text { dried } \\
\text { tealeaf } / \mathrm{yr}\end{array}$ & $0.07(0.01-0.47)$ \\
\hline 2009 [17] & Colorectal & 678 & $\begin{array}{l}\text { None vs. } \geq 750 \mathrm{~g} \text { dried } \\
\text { tealeaf/yr }\end{array}$ & $0.45(0.25-0.82)$ \\
\hline 2009 [17] & Leukemia & 117 & $\begin{array}{l}\text { None vs. } \geq 750 \mathrm{~g} \text { dried } \\
\text { tealeaf/yr }\end{array}$ & $0.57(0.07-4.79)$ \\
\hline
\end{tabular}

Table 2 summarizes the results of observational studies conducted by our research team. It shows the details of the year of the study's commencement, cancer site, number of participants, exposure level of green tea, ORs or HR, and their corresponding 95\% CIs by study, design, and 
location. An inverse association was observed consistently between green tea consumption, and risks of ovarian, breast, and colorectal cancers, and adult leukemia, with case mortality from ovarian cancer in our studies. In particular, adjusted HR and 95\% CI for case mortality from ovarian cancer was $0.40(0.18-0.90)$ in those who consumed green tea at the highest level compared with non-tea drinkers. Compared with never or seldom tea drinkers, the adjusted ORs and their corresponding $95 \%$ CIs were $0.43(0.30-0.63)$ for ovarian cancer, $0.61(0.48-0.78)$ for breast cancer in southeast China, 0.39(0.17-0.91) for adult leukemia, 0.43(0.21-0.91) for breast cancer in northeast China, and 0.45(0.25-0.82) for colorectal cancer. Significant inverse doseresponse relationships were also observed for quantity, duration, and frequency of green tea consumed.

\section{DISCUSSION:}

Tea is one of the most frequently consumed beverages worldwide. Green tea is known to contain tea polyphenols and other antioxidant compounds, which may be anti-carcinogenic, and extracts of tea have been shown to block nitrosamine-induced cancer in experimental animals [10,11]. As a possible cancer preventive agent, green tea has been the subject of considerable interest over the past 30 years. Laboratory studies based on in vitro and in vivo models have demonstrated anti-carcinogenic effects of tea polyphenols[11], and the inhibitory effects of green tea against carcinogenesis have been consistently revealed in a variety of organs in rodents[10]. In contrast, despite such clear indications from laboratory studies, the evidence on association between green tea drinking and human cancers has been mixed, with some studies finding green tea intake associated with reduced risk of cancer. Other studies however, have found no evidence of protective activity against certain human cancers[21]. Such inconsistency may be due to differences in tea types and the duration of habitual tea consumption in various study populations, or may have been due to lack of control of confounding factors[22]. A lack of detailed and specific information on tea consumption also limited the conclusions drawn from previous research.

A series of epidemiologic studies, specifically designed to evaluate the hypothesis that green tea protects against cancers, have been conducted by our research team over the past decade. From these cohort and case-control studies, we observed that green tea consumption has been consistently associated with a lower case mortality from ovarian cancer, and with decreased risks of incidence of ovarian, breast, and colorectal cancers, and leukemia in Chinese populations. Our findings are consistent with the evidence from experimental studies[10,11]. There have now been over 50 cohort and case-control studies of green tea and cancer incidence, including those conducted in China by our research team[12-14]. Our studies have been ranked in the highest quality group by a Cochrane review of green tea and cancer[21]. The case-control study of adult leukemia that we conducted in China found that green tea drinking reduced the risk of leukaemia occurrence[15]. This study was the first of its type, and other investigators have since obtained results confirming a preventive effect on incidence in Japan and Taiwan[23,24]. The finding is supported by an interventional study in patients with early stage chronic lymphocytic leukemia. The Mayo Clinic recently reported that EGCG intake correlated 
with reductions in absolute lymphocyte counts and degree of lymphadenopathy in a phase II trial [25].

In view of the variations in tea drinking practices in southeast and northeast China, the research team performed two case-control studies in northeast China. The studies conducted in northeast China adopted the same research methods established in southeast China, and in particular, used the same questionnaire to measure tea consumption to assess potential confounders. We report the characteristics of participants in breast cancer studies conducted in southeast China 2004-2005, and in northeast China 2008-2009 in Table 1. There were sizable variations in demographic characteristics between the participants in these two studies, including education, living in a rural area, tobacco smoking, alcohol drinking, physical activity, and energy intake. Particularly, there were a differences in tea consumption between the participants in southeast and northeast China. Despite the different distributions of tea drinking in these two Chinese populations, the apparent protective effects on breast cancer in both populations were of mostly the same magnitude.

Most studies yielding null results in this field have been conducted in western populations that exclusively consume black tea[26]. It is important to identify the potential confounders of green tea consumption in these and other study populations. Our studies found that green tea drinkers were more likely than non-tea drinker to reside in urban areas, have better education, and have a higher consumption of coffee, alcohol, soy, vegetables, and fruits[14]. Thus, any residual confounding from these lifestyle factors may affect the apparent protective effect of green tea.

Since the finding that green tea has a protective effect on cancers has important implications, it is necessary to emphasise the limitations and strengths of our work to date. A feature of our approach has been that extensive information was obtained on tea consumption as well as on diet, lifestyle, and factors relevant to hormonal status. A validated and reliable instrument specifically for Chinese populations was used to collect the information. Test-retest results confirmed the reproducibility of the questionnaire. Nevertheless, the case-control design may have introduced certain sources of bias. The cancer cases were identified from medical records, and interviewed in wards, while the controls were randomly selected from inpatients or outpatients in the same hospitals by matching the cases.

We conducted three case-control studies on leukemia, breast, and colorectal cancers in China between 2009 and 2010, using duplicate, parallel community, and hospital control groups that matched separately to the incident cases by age, gender, and residency. Demographic and lifestyle factors were measured and compared between the two control groups. We found that hospital controls were comparable with community controls for most demographic characteristics and lifestyle factors measured. It has been argued that due to the structures and processes of the health system in China, hospital outpatients provide a satisfactory control group in hospital-based case-control study in the Chinese hospital setting[17].

Our research interest on any type of tea consumption(not specifically green tea), and the cancers studied, were revealed at the time of the interview to participants to explain why the questions on tea were so detailed. However, in assessing the likelihood of any information bias which we consider to have been low, it was relevant that no mention occurred in popular media 
of any association between tea consumption and cancers prior to, and at the time of the research. Although tea consumption, like other personal habits, can be reported by the subjects with reasonable accuracy, misclassification of exposure may still exist. However, such errors are likely to bias results towards the null, and could not account for the strongly inverse associations reported here. Exposure of cases to risk factors may change due to disease status. However, the majority of cases were newly diagnosed and interviewed within three months. It appears unlikely that disease status materially affected the interview responses. The notion that cases would have either recalled more tea drinking or dramatically increased their tea drinking is implausible.

This summary report of six observational studies provides strong evidence that green tea consumption is associated with an improved survival of ovarian cancer, and a reduced risk of occurrence of certain cancers and adult leukaemia. We have almost completed three parallel case-control studies on breast and colorectal cancer, and adult leukemia with ascertainment of 16 genetic polymorphisms in an effort to illuminate molecular causal pathways. We have been conducting three placebo-controlled randomised clinical trials in Australia and China since 2009, using encapsulated green tea extract to assess the effects of green tea polyphenols in slowing leukaemia progression, and decreasing breast mammographic density and hormones levels in the peripheral blood of women who are at high risk of breast cancer. These on-going studies will provide further evidence on whether or not green tea has protective effects against cancers.

\section{CONCLUSION:}

In conclusion, our studies suggest that higher consumption of green tea enhances survival of ovarian cancer, and decreases the risks of ovarian, breast, and colorectal cancers, and adult leukemia. It follows that increased consumption of green tea in those having little or no consumption could protect against cancer development and progression. This finding has biological plausibility, and is supported by strong evidence of tumor-inhibitory effects of green tea in animal studies and other in-vitro and in-vivo experiments. More research to examine the nature of any causal relationship between green tea consumption and cancers is warranted. Evidence from our observational studies, which supports the protective effect of green tea against cancers, provides a knowledge platform from which to launch interventional studies for cancer prevention at the next stage. This logical and well justified next step has now commenced.

Abbreviations: Odds ratios (ORs), hazard ratios (HRs), 95\% confidence intervals (95\% CIs), epigallocatechin-3-gallate (EGCG), body mass index (BMI), International Federation of Gynecology and Obstetrics (FIGO)

Competing interests: The authors declare that they have no competing interests.

Acknowledgements and Funding: The authors acknowledge with gratitude the participation of patients, outpatients and community residents in China. We are grateful for the collaboration received from the participating hospitals, their staff, and from community councils. 
The studies were supported by the National Health and Medical Research Council (NHMRC) of Australia_project grant (ID 572542) and the Cancer Foundation of Western Australia project grant. The first author was supported from NHMRC postdoctoral fellowship (ID 303292). L Li was supported by the Australian Postgraduate Award and the University of Western Australia Establishment Award. P Liu was supported by the Scholarship for International Research Fees and University Postgraduate Award International Student.

\section{REFERENCES:}

1. Graham HN. Green tea composition, consumption, and polyphenol chemistry. Prev Med 1992; 21: 334-350.

2. Phipps RP. The second international scientific symposium on tea and human health. Nutr 1999; 15: 968-971.

3. Manach C, Williamson G, Morand C, Scalbert A, Remesy C. Bioavailability and bioefficacy of polyphenols in humans. Am J Clin Nutr 2005; 81: 230S-242S.

4. Yang CS, Lee MJ, Chen L. Human salivary tea catechin levels and catechin esterase activities: implication in human cancer prevention studies. Cancer Epidem Biomar 1999; 8: 83-89.

5. Suganuma M, Okabe S, Sueoka N, Sueoka E, Matsuyama S, Imai K, et al. Green tea and cancer chemoprevention. Mutat Res 1999; 428: 339-344.

6. Yang CS, Maliakal P, Meng X. Inhibition of carcinogenesis by tea. Annu Rev Pharmacol Toxicol 2002; 42: 25-54.

7. Hakim IA, Harris RB, Chow HH, Dean M, Brown S, Ali IU. Effect of a 4-month tea intervention on oxidative DNA damage among heavy smokers: role of glutathione Stransferase genotypes. Cancer Epidem Biomar 2004; 13: 242-249.

8. Luo H, Tang L, Tang M, Billam M, Huang T, Yu J, et al. Phase IIa chemoprevention trial of green tea polyphenols in high-risk individuals of liver cancer: modulation of urinary excretion of green tea polyphenols and 8-hydroxydeoxyguanosine. Carcinogenesis 2006; 27: 262-268.

9. Cabrera C, Artacho R, Giménez R. Beneficial effects of green tea--a review. J Am Coll Nutr 2006; 25: 79-99.

10. Chung FL, Schwartz J, Herzog CR, Yang YM. Tea and cancer prevention: studies in animals and humans. J Nutr 2003; 133: 3268S-74S.

11. Lambert JD, Hong J, Yang G, Liao J, Yang CS. Inhibition of carcinogenesis by polyphenols: evidence from laboratory investigation. Am J Clin Nutr 2005; 81: 284S91S.

12. Zhang M, Lee AH, Binns CW, Xie X. Green tea consumption enhances survival of epithelial ovarian cancer. Int J Cancer 2004; 112: 465-469.

13. Zhang M, Binns CW, Lee AH. Tea consumption and ovarian cancer risk: a case-control study in China. Cancer Epidem Biomar 2002; 11: 713-718.

14. Zhang M, Holman CDJ, Huang J-P, Xie X. Green tea and the prevention of breast cancer: a case-control study in Southeast China. Carcinogenesis 2007; 28: 1074-1078. 
15. Zhang M, Zhao XY, Zhang XD, Holman CDJ. Possible protective effect of green tea intake on risk of adult leukaemia. Brit J Cancer 2008; 98: 168-170.

16. Shi J, Zhang M, Li L, Holman CDJ, Chen J, Teng YE, Liu YP. Body mass index and its change in adulthood and breast cancer risk. Asian Pac J Cancer Prev 2010; 11: 12131218.

17. Li L, Zhang M, Holman CDJ. Population versus hospital controls for case-control studies on cancers in Chinese hospitals. BMC Med Res Methodol 2011; 11: 167, doi:10.1186/1471-2288-11-167.

18. Zhang M, Yang ZY, Binns CW, Lee AH. Diet and ovarian cancer risk: a case-control study in China. Brit J Cancer 2002; 86: 712-717.

19. Zhang M, Binns CW, Lee AH. Dietary patterns and nutrient intake of adult women in south-east China: a nutrition study in Zhejiang Province. Asia Pac J Clin Nutr 2002; 11 : 13-21.

20. Zhang M, Binns CW, Lee AH. A quantitative food frequency questionnaire for women in southeast China: Development and reproducibility. Asia Pac J Public Health 2005; 17: 29-34.

21. Boehm K, Borrelli F, Ernst E, Habacher G, Hung SK, Milazzo S, et al. Green tea (Camellia sinensis) for the prevention of cancer. Cochrane Database Syst Rev 2009; 8: CD005004.

22. Hakim IA, Harris RB, Weisgerber UM. Tea intake and squamous cell carcinoma of the skin: influence of type of tea beverages. Cancer Epidemiol Biomark Prev 2000; 9: 727731.

23. Kuo YC, Yu CL, Liu CY, Wang SF, Pan PC, Wu MT, et al. A population-based, casecontrol study of green tea consumption and leukemia risk in southwestern Taiwan. Cancer Causes Control 2009; 20: 57-65.

24. Naganuma T, Kuriyama S, Kakizaki M, Sone T, Nakaya N, Ohmori-Matsuda K, et al. Green tea consumption and hematologic malignancies in Japan: the Ohsaki study. Am J Epidemiol 2009; 170: 730-8.

25. Shanafelt TD, Call TG, Zent CS, Leis JF, Laplant B, Bowen DA et al. Phase 2 trial of daily, oral polyphenon $\mathrm{E}$ in patients with asymptomatic, Rai stage 0 to II chronic lymphocytic leukemia. Cancer 2012 Jul 3. doi: 10.1002/cncr.27719.

26. Sun CL, Yuan JM, Koh WP, Yu MC. Green tea, black tea and breast cancer risk: a metaanalysis of epidemiological studies. Carcinogenesis 2006; 27: 1310-5 